\title{
Present status of water mite species (Acari, Hydrachnidia) described by Motaş, Tanasachi and Orghidan from Romania
}

\author{
Mirela CîMPEAN, ${ }^{1}$ KARINA PAUla BATTES ${ }^{2}$
}

Babeş-Bolyai University, Faculty of Biology and Geology, Department of Taxonomy and Ecology, 5-7 Clinicilor Street, 400006, Cluj-Napoca, Romania, e-mail: mirela.cimpean@ubbcluj.ro

2 Babeş-Bolyai University, Faculty of Biology and Geology, Department of Taxonomy and Ecology, 5-7 Clinicilor Street, 400006, Cluj-Napoca, Romania, e-mail: karina.battes@ubbcluj.ro

Keywords $\quad$ synonym species, valid species, species dubia, species incertae

Abstract The present paper investigates the validity of the water mite species (Acari, Hydrachnidia) described from Romania by Motaş, Tanasachi and Orghidan. The Romanian researchers from the "Emil Racoviţă" Speleology Institute, Romanian Academy, Bucharest, had a prolific activity between 1939 and 1963: among the taxa new to science described by these authors, 34 species and 3 subspecies had their type localities in Romania. More than 50 years later, 23 species and one subspecie described by the team coordinated by Motaş are still valid. 17 of these taxa were found all over Europe, while 7 were not recorded outside Romania until present day.

\section{Obecny status wodopójek (Acari, Hydrachnidia) z Rumunii opisanych przez Motaşa, Tanasachiego i Orghidana}

Słowa kluczowe gatunki synonimiczne, gatunki obowiązujące, gatunki wątpliwe, gatunki niepewne

Streszczenie

\begin{abstract}
W niniejszym artykule zbadano aktualność gatunków wodopójek (Acari, Hydrachnidia) opisanych z terenu Rumunii przez Motaşa, Tanasachi i Orghidana. Główna aktywność rumuńskich badaczy z Instytutu Speleologii "Emil Racoviţă”, Akademii Rumuńskiej w Bukareszcie, przypadała na lata 1939-1963. Wśród nowych gatunków opisywanych przez tych autorów locus typicus 34 gatunków i 3 podgatunków znajdowały się na terenie Rumunii. Ponad 50 lat później, 23 gatunki i jeden podgatunek opisane przez zespół prowadzony przez Motaşa są nadal aktualne. $17 \mathrm{z}$ tych taksonów odnotowano na terenie całej Europy, a 7 innych do dzisiaj stwierdzonych jest jedynie na terenie Rumunii.
\end{abstract}

\section{Introduction}

Hydrachnidia (the water mites), also called Hydrachnellae or Hydracarina, represents the most important group of freshwater Arachnida, with more than 6,000 species described worldwide (Di Sabatino et al., 2008). The Palaearctic region is one of the best investigated areas, with the highest number of species recorded (1,642 species) (Di Sabatino et al., 2008). 
The first Romanian study on water mites (Acari, Hydrachnidia) was conducted in 1923, when Motaş published „Contribution à l'étude des Acariens d'eau douce de Roumanie” (Motaş, 1923). More than 70 scientific papers were published between 1923 and 1972 by Motaş, Tanasachi and Orghidan, dealing with water mites from different environments: rivers, lakes and interstitial habitats.

In 1979, Konnerth-Ionescu published an inventory of all water mite species from Romania, with their exact locations. 267 water mite species and 18 subspecies were listed, from 367 sampling locations considered until then (Konnerth-Ionescu, 1979).

An updated and complemented list of water mite species from Romania was published in recent years. It included 251 species recognized at present in Romania (Cîmpean, 2011).

\section{Discussions}

Between 1939 and 1963, Constantin Motaş, Jeanne Tanasachi (Şoarec before marriage) and Traian Orghidan described 34 species and 3 subspecies new to science in Romania (Table 1) (Motaş, Şoarec, 1939; Şoarec, 1939; Motaş, 1940, 1959; Motaş, Tanasachi, 1944, 1946, 1948a, 1948b, 1960, 1963; Motaş et al., 1946, 1947a, 1947b, 1947c, 1957a, 1957b, 1958; Tanasachi, Orghidan, 1955).

23 water mite species and one subspecie from those described by the team coordinated by Motaş are still valid, according to the Hydrachnidia taxonomic revisions made at an European scale (Table 1).

Table 1 Water mite species new to science, described by Motaş and his team (the current status, the type localities, the European distribution and the preferred habitats)

\begin{tabular}{|c|c|c|c|c|c|}
\hline No. & Taxa & Species status & Type localities & Distribution & Habitat \\
\hline 1 & 2 & 3 & 4 & 5 & 6 \\
\hline 1. & $\begin{array}{l}\text { Stygothrombium } \\
\text { racovitzai (Motaş, } \\
\text { Tanasachi, 1946) }\end{array}$ & $\begin{array}{l}\text { Species dubia } \\
\text { (Davids et al., } \\
\text { 2007). } \\
\text { Future material } \\
\text { from the Romanian } \\
\text { populations is } \\
\text { necessary. }\end{array}$ & $\begin{array}{l}\text { The Crișul Repede } \\
\text { River in Șuncuiuș } \\
\text { village (Motaş, } \\
\text { Tanasachi, 1946) }\end{array}$ & - & - \\
\hline 2. & $\begin{array}{l}\text { Tadjikothyas fibulata } \\
\text { (Motaş, Tanasachi, } \\
\text { 1957) }\end{array}$ & Valid species & $\begin{array}{l}\text { Rheocren spring } \\
\text { tributary of Lake } \\
\text { Greaca (drained) } \\
\text { (Motaş et al., 1957b) }\end{array}$ & Limnofauna: $12, \mathrm{Y}^{*}$ & $\begin{array}{l}\text { Crenophylous } \\
\text { (springs and small } \\
\text { rivers) (Motaş et } \\
\text { al., 1957b; Motaş, } \\
\text { 1959) }\end{array}$ \\
\hline 3. & $\begin{array}{l}\text { Dacothyas } \\
\text { savulescui (Motaş, } \\
1959)\end{array}$ & Valid species & $\begin{array}{l}\text { Spring in Comana } \\
\text { village, } 30 \mathrm{~km} \text { south } \\
\text { from Bucharest } \\
\text { (Motaş, 1959) }\end{array}$ & $\begin{array}{l}\text { Limnofauna: } 12^{*} \\
\text { Romania }\end{array}$ & $\begin{array}{l}\text { Crenobiont } \\
\text { (springs) (Motaş, } \\
1959 \text { ) }\end{array}$ \\
\hline 4. & $\begin{array}{l}\text { Vietsthyas fonticola } \\
\text { (Motaş, Tanasachi, } \\
\text { 1957) }\end{array}$ & $\begin{array}{l}\text { A junior synonym } \\
\text { to Tartarothyas } \\
\text { micrommata } \text { Viets, } \\
1934\end{array}$ & - & - & - \\
\hline
\end{tabular}




\begin{tabular}{|c|c|c|c|c|c|}
\hline 1 & 2 & 3 & 4 & 5 & 6 \\
\hline 5. & $\begin{array}{l}\text { Lebertia holsatica } \\
\text { nitida (Motaş, } \\
\text { Tanasachi, 1963) }\end{array}$ & $\begin{array}{l}\text { Non valid } \\
\text { subspecies } \\
\text { This subspecies } \\
\text { is insufficiently } \\
\text { defined and } \\
\text { agree with } \\
\text { L. semireticulata } \\
\text { Viets, } 1925 \\
\text { (Gerecke, 2009; } \\
\text { Di Sabatino et al., } \\
\text { 2010) }\end{array}$ & - & - & - \\
\hline 6. & $\begin{array}{l}\text { Torrenticola jeanneli } \\
\text { (Motaş, Tanasachi, } \\
\text { 1947) }\end{array}$ & Valid species & $\begin{array}{l}\text { The Târlungul } \\
\text { Stream (near } \\
\text { Satul-Lung village, } \\
\text { Brașov county); } \\
\text { The Bîrsa Stream, } \\
\text { tributary of the Olt } \\
\text { River; The Cerna } \\
\text { River (Motaș et al., } \\
\text { 1947b) }\end{array}$ & $\begin{array}{l}\text { Limnofauna: } 4,5, \\
7,10^{*} \\
\text { Central and } \\
\text { Southeastern } \\
\text { Europe. Only known } \\
\text { from a few sites } \\
\text { (Di Sabatino et al., } \\
\text { 2010) }\end{array}$ & $\begin{array}{l}\text { Low- and middle } \\
\text { order streams, } \\
\text { only known from } \\
\text { hyporheic }\end{array}$ \\
\hline 7. & $\begin{array}{l}\text { Kawamuracarus } \\
\text { chappuisi (Motaş, } \\
\text { Tanasachi, 1946) }\end{array}$ & $\begin{array}{l}\text { Valid species } \\
K . \text { chappuisi was } \\
\text { proposed to be } \\
\text { a synonym of } \\
\text { K. vardaricolus } \\
\text { Viets, } 1942 . \\
\text { A resolution is } \\
\text { hampered by the } \\
\text { loss of type material } \\
\text { of } K . \text { chappuisi } \\
\text { (Gerecke et al., } \\
\text { 2016). Future } \\
\text { material from } \\
\text { the Romanian } \\
\text { populations } \\
\text { could solve this } \\
\text { discussion. }\end{array}$ & $\begin{array}{l}\text { The Valea } \\
\text { Drăganului Stream, } \\
\text { tributary of the } \\
\text { Crișul Repede River } \\
\text { (Bihor county) } \\
\text { (Motaş, Tanasachi, } \\
\text { 1946) }\end{array}$ & $\begin{array}{l}\text { Limnofauna: } 10^{*} \\
\text { Southeastern and } \\
\text { eastern Europe, } \\
\text { one record from the } \\
\text { eastern part of the } \\
\text { area covered, Poland } \\
\text { (Gerecke et al., 2016) }\end{array}$ & $\begin{array}{l}\text { Interstitial waters } \\
\text { of low- and middle } \\
\text { order streams }\end{array}$ \\
\hline 8. & $\begin{array}{l}\text { Atractides latipalpis } \\
\text { (Motaş, Tanasachi, } \\
\text { 1946) }\end{array}$ & Valid species & $\begin{array}{l}\text { The Valea } \\
\text { Drăganului Stream, } \\
\text { tributary of the } \\
\text { Crișul Repede River } \\
\text { (Bihor county) } \\
\text { (Motaş, Tanasachi, } \\
\text { 1946) }\end{array}$ & $\begin{array}{l}\text { Limnofauna: } 2,4,5, \\
7,8,9,10,11,13,18^{*} \\
\text { Central and } \\
\text { southeastern Europe, } \\
\text { United Kingdom } \\
\text { (Di Sabatino et al., } \\
\text { 2010) }\end{array}$ & Hyporheobiont \\
\hline
\end{tabular}




\begin{tabular}{|c|c|c|c|c|c|}
\hline 1 & 2 & 3 & 4 & 5 & 6 \\
\hline 9. & $\begin{array}{l}\text { Atractides } \\
\text { magnirostris (Motaş, } \\
\text { Tanasachi, 1948) }\end{array}$ & $\begin{array}{l}\text { Species dubia } \\
\text { Possibly synonym } \\
\text { to } \text { A. acutirostris } \\
\text { (Motas, Angelier, } \\
\text { 1927) (Gerecke, } \\
\text { 2003) }\end{array}$ & $\begin{array}{l}\text { The Sadul Stream, } \\
\text { right tributary of } \\
\text { the Cibin River (the } \\
\text { Olt River catchment } \\
\text { area); the Bughea } \\
\text { Stream (in Câmpul- } \\
\text { Lung), tributary of } \\
\text { the Rîl Târgului } \\
\text { River (the Argeș } \\
\text { River catchment } \\
\text { area) (Motaş, } \\
\text { Tanasachi, 1948b) }\end{array}$ & - & - \\
\hline 10. & $\begin{array}{l}\text { Atractides } \\
\text { microphtalmus } \\
\text { (Motaş, Tanasachi, } \\
\text { 1948) }\end{array}$ & \begin{tabular}{|l|} 
Non valid species \\
Species similar \\
with A. denticulatus \\
(Walter, 1947) and \\
possible also with \\
other species of \\
the cisternarum- \\
species group \\
(Di Sabatino et al., \\
2010). Further \\
investigations on \\
the geographical \\
variation in species \\
of this group are \\
necessary (Gerecke, \\
2003) \\
\end{tabular} & - & - & - \\
\hline 11. & $\begin{array}{l}\text { Atractides nodipalpis } \\
\text { intermedius (Şoarec, } \\
\text { 1939) }\end{array}$ & $\begin{array}{l}\text { Subspecies dubia } \\
\text { (Gerecke, 2003) }\end{array}$ & $\begin{array}{l}\text { The Secu Stream, } \\
\text { tributary of the } \\
\text { Ozana River } \\
\text { (the Siret River } \\
\text { catchment area) } \\
\text { (Șoarec, 1939) }\end{array}$ & - & - \\
\hline 12. & $\begin{array}{l}\text { Atractides } \\
\text { phreaticus (Motaş, } \\
\text { Tanasachi, 1948) }\end{array}$ & Valid species & $\begin{array}{l}\text { The Rîul Mare } \\
\text { Stream, left } \\
\text { tributary of the } \\
\text { Olt River (near } \\
\text { Porumbacul de } \\
\text { Sus village); the } \\
\text { Sebeș Stream, } \\
\text { left tributary of } \\
\text { the Olt River; the } \\
\text { Bogata Stream, } \\
\text { left tributary of the } \\
\text { Olt River (Motas, } \\
\text { Tanasachi, 1948b) }\end{array}$ & $\begin{array}{l}\text { Limnofauna: } 4,7,8, \\
9,10^{*} \\
\text { Central and } \\
\text { southeastern Europe, } \\
\text { southern France } \\
\text { (Di Sabatino et al., } \\
\text { 2010) }\end{array}$ & Hyporheobiont \\
\hline 13. & $\begin{array}{l}\text { Atractides prosiliens } \\
\text { (Motaş, Tanasachi, } \\
\text { 1948) }\end{array}$ & Valid species & $\begin{array}{l}\text { The Bughea Stream, } \\
\text { tributary of the Rîul } \\
\text { Târgului River (the } \\
\text { Argess catchment } \\
\text { area) (the Southern } \\
\text { Carpathians) } \\
\text { (Motass, Tanasachi, } \\
\text { 1948b) }\end{array}$ & $\begin{array}{l}\text { Limnofauna: } 10^{*} \\
\text { Romania, doubtful } \\
\text { records from Austria } \\
\text { and southern France } \\
\text { (Di Sabatino et al., } \\
\text { 2010) }\end{array}$ & Hyporheobiont \\
\hline
\end{tabular}




\begin{tabular}{|c|c|c|c|c|c|}
\hline 1 & 2 & 3 & 4 & 5 & 6 \\
\hline 14. & $\begin{array}{l}\text { Atractides pygmaeus } \\
\text { (Motaş, Tanasachi, } \\
\text { 1948) }\end{array}$ & Valid species & $\begin{array}{l}\text { The Rîuşor Stream, } \\
\text { tributary of the } \\
\text { Dâmbovița River } \\
\text { (the Southern } \\
\text { Carpathians) } \\
\text { (Motaş, Tanasachi, } \\
\text { 1948b) }\end{array}$ & $\begin{array}{l}\text { Limnofauna: } 3,4,5 \text {, } \\
\text { 7, } 10^{*} \\
\text { the Alps, the } \\
\text { Carpathians, } \\
\text { Montenegro } \\
\text { (Di Sabatino et al., } \\
\text { 2010) }\end{array}$ & Hyporheobiont \\
\hline 15. & $\begin{array}{l}\text { Atractides sokolowi } \\
\text { (Motaş, Tanasachi, } \\
\text { 1948) }\end{array}$ & Valid species & $\begin{array}{l}\text { The Bughea Stream, } \\
\text { tributary of the Rîul } \\
\text { Târgului River (the } \\
\text { Argeș catchment } \\
\text { area) (the Southern } \\
\text { Carpathians) } \\
\text { (Motaş, Tanasachi, } \\
\text { 1948b) }\end{array}$ & $\begin{array}{l}\text { Limnofauna: } 7,10^{*} \\
\text { Eastern and } \\
\text { southeastern Europe; } \\
\text { from area covered } \\
\text { recorded in Poland } \\
\text { (Di Sabatino et al., } \\
2010 \text { ) }\end{array}$ & Rhithrobiont \\
\hline 16. & $\begin{array}{l}\text { Atractides szalay } \\
\text { (Motaş, Tanasachi, } \\
\text { 1948) }\end{array}$ & $\begin{array}{l}\text { A junior synonym } \\
\text { to } A \text {. oblongus } \\
\text { (Walter, 1944) }\end{array}$ & - & - & - \\
\hline 17. & $\begin{array}{l}\text { Atractides elegans } \\
\text { (Motaş, Tanasachi, } \\
\text { 1948) }\end{array}$ & $\begin{array}{l}\text { Species incertae } \\
\text { Species similar with } \\
\text { A. orghidani Motaş } \\
\& \text { Tanasachi, } 1960 \\
\text { (Di Sabatino et al., } \\
\text { 2010). }\end{array}$ & $\begin{array}{l}\text { The Sadul Stream, } \\
\text { right tributary of } \\
\text { the Cibin River (the } \\
\text { Olt River catchment } \\
\text { area) (Motaş, } \\
\text { Tanasachi, 1948b) }\end{array}$ & - & - \\
\hline 18. & $\begin{array}{l}\text { Atractides orghidani } \\
\text { (Motaş, Tanasachi, } \\
\text { 1960) }\end{array}$ & Valid species & $\begin{array}{l}\text { The Vîrghiș Stream, } \\
\text { tributary of the Olt } \\
\text { River in Merești } \\
\text { village (Harghita } \\
\text { county) (Motaş, } \\
\text { Tanasachi, 1960) }\end{array}$ & $\begin{array}{l}\text { Limnofauna: } 3,5,6, \\
\text { 9, 10, 13, Y* } \\
\text { Romania, Greece, } \\
\text { Montenegro, Italy, } \\
\text { France, Turkey } \\
\text { (Di Sabatino et al., } \\
\text { 2010) }\end{array}$ & $\begin{array}{l}\text { Rhithrobiont, } \\
\text { hyporheophilous }\end{array}$ \\
\hline 19. & $\begin{array}{l}\text { Feltria mira (Motaş, } \\
\text { Tanasachi, 1948) }\end{array}$ & $\begin{array}{l}\text { Species incertae } \\
\text { Species similar } \\
\text { with } F \text {. motasi } \\
\text { (Schwoerbel, 1961; } \\
\text { Gerecke, 2012; } \\
\text { Gerecke et al., 2016) }\end{array}$ & $\begin{array}{l}\text { The Prahova River } \\
\text { (near Azuga city) } \\
\text { (Motaş, Tanasachi, } \\
\text { 1948a) }\end{array}$ & - & - \\
\hline 20. & $\begin{array}{l}\text { Feltria amplexa } \\
\text { (Motaş, Tanasachi, } \\
\text { 1944) }\end{array}$ & $\begin{array}{l}\text { Valid species } \\
\text { (Further } \\
\text { investigations are } \\
\text { necessary) }\end{array}$ & $\begin{array}{l}\text { Rheocren spring, } \\
\text { right tributary of the } \\
\text { Valea Rea Stream in } \\
\text { Sinaia city (Motaş, } \\
\text { Tanasachi, 1944) } \\
\end{array}$ & Limnofauna: $10^{*}$ & - \\
\hline 21. & $\begin{array}{l}\text { Feltria halberti } \\
\text { (Motaş, Tanasachi, } \\
\text { 1957) }\end{array}$ & $\begin{array}{l}\text { A junior synonym } \\
\text { to } F \text {. rouxi (Walter, } \\
1907 \text { ) }\end{array}$ & - & - & - \\
\hline 22. & $\begin{array}{l}\text { Feltria simionescui } \\
\text { (Motaş, Soarec, } \\
\text { 1939) }\end{array}$ & $\begin{array}{l}\text { A junior synonym } \\
\text { to } F . \text { zschokkei } \\
\text { (Koenike, 1896) }\end{array}$ & - & - & - \\
\hline 23. & $\begin{array}{l}\text { Forelia aspidiophora } \\
\text { (Motaş, 1959) }\end{array}$ & $\begin{array}{l}\text { Valid species } \\
\text { (Further } \\
\text { investigations are } \\
\text { necessary) }\end{array}$ & $\begin{array}{l}\text { The Cocora Stream, } \\
\text { left tributary of the } \\
\text { Ialomița River, near } \\
\text { Peștera village (the } \\
\text { Bucegi Mountains) } \\
\text { (Motaș, 1959) }\end{array}$ & Limnofauna: $10^{*}$ & - \\
\hline
\end{tabular}




\begin{tabular}{|c|c|c|c|c|c|}
\hline 1 & 2 & 3 & 4 & 5 & 6 \\
\hline 24. & $\begin{array}{l}\text { Albaxona lundbladi } \\
\text { (Motaş, Tanasachi, } \\
\text { 1947) }\end{array}$ & Valid species & $\begin{array}{l}\text { The Bogata Stream, } \\
\text { tributary of the Olt } \\
\text { River (the Southern } \\
\text { Carpathians) (Motaș } \\
\text { et al., 1947b) }\end{array}$ & $\begin{array}{l}\text { Limnofauna: } 3,4,5, \\
7,8,9,10,18, Y^{*} \\
\text { British Isles, central, } \\
\text { southern and } \\
\text { southeastern Europe } \\
\text { (Gerecke et al., 2016) }\end{array}$ & Hyporheobiont \\
\hline 25. & $\begin{array}{l}\text { Paraxonopsis } \\
\text { inferorum (Motaş, } \\
\text { Tanasachi, 1947) }\end{array}$ & Valid species & $\begin{array}{l}\text { The Târlungul } \\
\text { Stream (near } \\
\text { Satul-Lung village, } \\
\text { Braşov county) } \\
\text { (Motaș et al., 1947b) } \\
\end{array}$ & $\begin{array}{l}\text { Limnofauna: } 2,3,4, \\
5,6,9,10,11,14,15^{*} \\
\text { Central, eastern and } \\
\text { southern Europe } \\
\text { (Gerecke et al., 2016) }\end{array}$ & Hyporheobiont \\
\hline 26. & $\begin{array}{l}\text { Paraxonopsis vietsi } \\
\text { (Motaş, Tanasachi, } \\
\text { 1947) }\end{array}$ & Valid species & $\begin{array}{l}\text { The Cerna River } \\
\text { (Herculane) } \\
\text { (Motaş et al., 1947b) }\end{array}$ & $\begin{array}{l}\text { Limnofauna: } 2,3,7, \\
10,12^{*} \\
\text { Central and southern } \\
\text { Europe (Gerecke } \\
\text { et al., 2016) }\end{array}$ & Hyporheobiont \\
\hline 27. & $\begin{array}{l}\text { Erebaxonopsis } \\
\text { brevipes (Motaş, } \\
\text { Tanasachi, 1947) }\end{array}$ & Valid species & $\begin{array}{l}\text { The Târlungul } \\
\text { Stream (near } \\
\text { Satul-Lung village, } \\
\text { Braşov county); } \\
\text { The Cerna River } \\
\text { (Herculane) } \\
\text { (Motaș et al., 1947b) }\end{array}$ & $\begin{array}{l}\text { Limnofauna: } 1,3,10^{*} \\
\text { SW Palearctic } \\
\text { (Gerecke et al., 2016) }\end{array}$ & Hyporheobiont \\
\hline 28. & $\begin{array}{l}\text { Aturus paucisetus } \\
\text { (Motaş, Tanasachi, } \\
\text { 1946) }\end{array}$ & $\begin{array}{l}\text { Valid species } \\
\text { Future material } \\
\text { from the Romanian } \\
\text { population is } \\
\text { necessary, because } \\
\text { after the loss of } \\
\text { the holotype, } \\
\text { morfological data } \\
\text { are mostly based on } \\
\text { central European } \\
\text { material (Gerecke } \\
\text { et al., 2016) }\end{array}$ & $\begin{array}{l}\text { The Crișul Repede } \\
\text { River in Șuncuiuș } \\
\text { village (Motaş, } \\
\text { Tanasachi, 1946) }\end{array}$ & $\begin{array}{l}\text { Limnofauna: } 2,4, \\
7,10^{*} \\
\text { Central and } \\
\text { southeastern Europe } \\
\text { (Gerecke et al., 2016) }\end{array}$ & $\begin{array}{l}\text { Low and middle } \\
\text { order streams, } \\
\text { preferably in } \\
\text { the hyporheic } \\
\text { (Gerecke et al., } \\
2016)\end{array}$ \\
\hline 29. & $\begin{array}{l}\text { Kongsbergia dentate } \\
\text { folioligera (Motaş, } \\
\text { Tanasachi, 1958) }\end{array}$ & $\begin{array}{l}\text { Valid subspecies } \\
\text { (Further } \\
\text { investigations are } \\
\text { necessary) }\end{array}$ & $\begin{array}{l}\text { The Salătruc } \\
\text { Stream, tributary of } \\
\text { the Topolog River } \\
\text { (Motaș et al., 1958) }\end{array}$ & Limnofauna: $10^{*}$ & - \\
\hline 30. & $\begin{array}{l}\text { Kongsbergia } \\
\text { d-motasi (Motaş, } \\
\text { Tanasachi, 1958) }\end{array}$ & $\begin{array}{l}\text { Valid species } \\
\text { (Further } \\
\text { investigations are } \\
\text { necessary) }\end{array}$ & $\begin{array}{l}\text { The Salătruc } \\
\text { Stream, tributary of } \\
\text { the Topolog River; } \\
\text { the Rîul Mare } \\
\text { Stream, tributary of } \\
\text { the Olt River (Motaș } \\
\text { et al., 1958) }\end{array}$ & Limnofauna: $7,10^{*}$ & - \\
\hline 31. & $\begin{array}{l}\text { Kongsbergia } \\
\text { pectinigera (Motaş, } \\
\text { Tanasachi, 1946) }\end{array}$ & Valid species & $\begin{array}{l}\text { The Valea } \\
\text { Drăganului Stream } \\
\text { and the Crișul } \\
\text { Repede River } \\
\text { (Bihor county) } \\
\text { (Motaş, Tanasachi, } \\
\text { 1946) }\end{array}$ & $\begin{array}{l}\text { Limnofauna: } 1,3,4 \text {, } \\
5,7,8,9,10^{*} \\
\text { Southern Europe, } \\
\text { south-central and } \\
\text { eastern Europe } \\
\text { (Gerecke et al., 2016) }\end{array}$ & Hyporheobiont \\
\hline
\end{tabular}




\begin{tabular}{|c|c|c|c|c|c|}
\hline 1 & 2 & 3 & 4 & 5 & 6 \\
\hline 32. & $\begin{array}{l}\text { Mideopsis fonticola } \\
\text { (Tanasachi, } \\
\text { Orghidan, 1955) }\end{array}$ & $\begin{array}{l}\text { Species incertae } \\
\text { Species similar with } \\
\text { Nudomideopsis } \\
\text { latipalpis } \\
\text { E. Angelier, } 1963 \\
\text { (Gerecke et al., } \\
\text { 2016) }\end{array}$ & $\begin{array}{l}\text { Olguța Gabor's } \\
\text { fountain from Ponor } \\
\text { village (Hunedoara } \\
\text { county) (Tanasachi, } \\
\text { Orghidan, 1955) }\end{array}$ & - & - \\
\hline 33. & $\begin{array}{l}\text { Bogatia maxillaris } \\
\text { (Motaş, Tanasachi, } \\
\text { 1948) }\end{array}$ & Valid species & $\begin{array}{l}\text { The Bogata Stream, } \\
\text { left tributary of the } \\
\text { Olt River (close to } \\
\text { Bogata village) } \\
\text { (Motaş, Tanasachi, } \\
\text { 1948a) }\end{array}$ & $\begin{array}{l}\text { Limnofauna: } 3,6^{*} \\
\text { Romania, Macedonia } \\
\text { and Sicily (Gerecke } \\
\text { et al., 2016) }\end{array}$ & Hyporheobiont \\
\hline 34. & $\begin{array}{l}\text { Chappuisides } \\
\text { thienemanni (Motaş, } \\
\text { 1959) }\end{array}$ & $\begin{array}{l}\text { Valid species } \\
\text { (Further } \\
\text { investigations are } \\
\text { necessary) }\end{array}$ & $\begin{array}{l}\text { The Bogata Stream, } \\
\text { tributary of the Olt } \\
\text { River; the Rîușorul } \\
\text { Stream, tributary } \\
\text { of the Dîmbovița } \\
\text { River, near } \\
\text { Cîmpulung Muscel; } \\
\text { the Dopca Stream, } \\
\text { left tributary of } \\
\text { the Olt River; the } \\
\text { Valea Drăganului } \\
\text { River (the Apuseni } \\
\text { Mountains); the } \\
\text { Valea Sighiștelului } \\
\text { Stream (the Apuseni } \\
\text { Mountains) (Motaș, } \\
\text { 1959) }\end{array}$ & Limnofauna: $10^{*}$ & - \\
\hline 35. & $\begin{array}{l}\text { Phreatohydracarus } \\
\text { mosticus (Tanasachi, } \\
\text { Orghidan, 1955) }\end{array}$ & Valid species & $\begin{array}{l}\text { Wells from Ponor } \\
\text { village (Hunedoara } \\
\text { county) (Tanasachi, } \\
\text { Orghidan, 1955) } \\
\end{array}$ & $\begin{array}{l}\text { Limnofauna: } 10^{*} \\
\text { Romania }\end{array}$ & Hyporheobiont \\
\hline 36. & $\begin{array}{l}\text { Arrenurus iassiensis } \\
\text { (Motaş, 1940) }\end{array}$ & $\begin{array}{l}\text { Valid species } \\
\text { (Further } \\
\text { investigations are } \\
\text { necessary) }\end{array}$ & $\begin{array}{l}\text { Pond on the right } \\
\text { bank of the Bahlui } \\
\text { River, near Iași } \\
\text { (Motaș, 1940) }\end{array}$ & Limnofauna: $16^{*}$ & - \\
\hline 37. & $\begin{array}{l}\text { Arrenurus } \\
\text { lundbladianus } \\
\text { (Motaş, Tanasachi, } \\
\text { 1958) }\end{array}$ & $\begin{array}{l}\text { A junior synonym } \\
\text { to } A \text {. corsicus } \\
\text { (E. Angelier, 1951) }\end{array}$ & - & - & - \\
\hline
\end{tabular}

* Information from the database based on data published by Viets (1978) in Limnofauna Europaea, updated in 1.09.2016 and published on watermite.org (2017) (1: Iberian Peninsula, 2: The Pyrenees, 3: Italy, 4: The Alps, 5: Dinaric Western Balkan, 6: Hellenic Western Balkan, 7: Eastern Balkan, 8: Western Highlands, 9: Central Highlands, 10: The Carpathians, 11: Hungarian Lowlands, 12: Pontic Province, 13: Western Lowlands, 14: Central Lowlands, 15: Baltic Province, 16: Eastern Lowlands, 18: Great Britan, Y: Israel, Turkey, Syria).

However, 6 out of the 24 valid taxa need further investigations, since holotype and paratype specimens are missing: Feltria amplexa (Motaş, Tanasachi, 1944), Forelia aspidiophora (Motaş, 1959), Kongsbergia dentate folioligera (Motaş, Tanasachi, 1958), Kongsbergia d-motasi (Motaş, Tanasachi, 1958), Chappuisides thienemanni (Motaş, 1959), Arrenurus iassiensis (Motaş, 
1940). These species were not classified during the latest European revision (Davids et al., 2007; Di Sabatino et al., 2010; Gerecke et al., 2016), but they are still considered valid in the databases, according to information published by K.O. Viets (1978), updated in 1.09.2016 and posted on watermite.org (2017).

Table 1 depicts updated information on the European distribution of valid water mite species described by the team coordinated by Motaş, together with the habitats they inhabit and the type localities they were described from.

Recent studies reported the following species in Romania: Kawamuracarus chappuisi and Aturus paucisetus in the Crişul Repede River (Pavelescu, Cîmpean, 2002-2003); Phreatohydracarus mosticus and Atractides cf. latipalpis in the Arieş River (Moldovan et al., 2011); Torrenticola jeanneli in the Someşul Cald River (Cîmpean, 2011).

The number of new species described by the team coordinated by Motaş versus the number of valid species during the 24 years (1939-1963) is depicted in Figure 1. The highest number of new described species was in 1948 (10), with 5 valid at present.

Four water mite species were proposed as candidates for the European Red List: Tadjikothyas fibulata, Kawamuracarus chappuisi, Chappuisides thienemanni and Phreatohydracarus mosticus (Cîmpean, 2014). Two species might need protection and could join the list, due to their restricted distribution: Dacothyas savulescui and Atractides prosiliens.

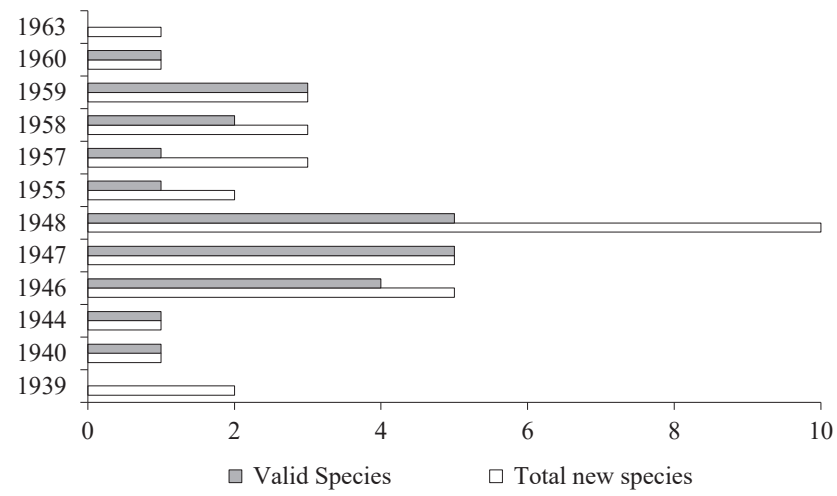

Figure 1. Total new versus valid water mite species (Acari, Hydrachnidia) described by Motaş, Tanasachi and Orghidan from Romania

Since several holotype and paratype specimens are missing, further investigations are mandatory for some water mite species, by revisiting the sampling regions.

\section{Acknowledgements}

We are thankful to Dr Reinhard Gerecke (Germany) and one anonymous referee for their careful work and valuable suggestions. 


\section{References}

Cîmpean, M. (2011). Studiul taxonomic şi ecologic asupra comunităţilor de acarieni acvatici (Acari, Hydrachnidia) din bazinul de drenaj al râului Someşul Mic şi rolul acestor organisme ca indicatori ai calităţii apei. Presa Universitară Clujeană.

Cîmpean, M. (2014). A review of the water mites (Acari, Hydrachnidia) from protected areas of Romania. North-western Journal of Zoology, 10 (Suppl. 1), 67-77.

Davids, C., Di Sabatino, A., Gerecke, R, Gledhill, T., Smit, H., Van Der Hammen H. (2007). Acari: Hydrachnidia. In: R. Gerecke (ed.), Süßwasserfauna Mitteleuropas, 7/2-1, Chelicerata, Acari I (pp. 241376). München: Spektrum Elsevier.

Di Sabatino, A., Smit, H., Gerecke, R., Goldschmidt, T., Matsumoto, N., Cicolani, B. (2008). Global diversity of water mites (Acari, Hydrachnidia; Arachnida) in freshwater. Hydrobiologia, 595, 303-315.

Di Sabatino, A., Gerecke, R., Gledhill T., Smit, H. (2010). Chelicerata: Acari II. Süßwasserfauna von Mitteleuropa, 7/2-2 (I-IX), 1-134.

Gerecke, R. (2003). Water mites of the genus Atractides Koch, 1837 (Acari: Parasitengona: Hygrobatidae) in the western Palaearctic region: A revision. Zoological Journal of the Linnean Society, 138 (2-3), $141-378$

Gerecke, R. (2009). Revisional studies on the European species of the water mite genus Lebertia Neuman, 1880 (Acari: Hydrachnidia: Lebertiidae). Abhandlungen der Senckenberg Gesellschaft für Naturforschung, 566, 1-144.

Gerecke, R. (2012). Studies on European Feltria species (Acari: Hydrachnidia: Feltriidae). Stuttgarter Beiträge zur Naturkunde A, Neue Serie 5, 13-47.

Gerecke, R., Gledhill, T., Pešić, V., Smit, H. (2016). Chelicerata: Acari III. Süßwasserfauna von Mitteleuropa, 7 (2-3), 1-429.

Konnerth-Ionescu, A. (1979). Conspectus des Hydrachnelles (Acari) de la Roumanie. Travaux du Museum d'Histoire Naturelle «Grigore Antipa», 20, 85-120.

Moldovan, O.T., Levei, E., Banciu, M., Banciu, H.L., Marin, C., Pavelescu, C., Brad, T., Cîmpean, M., Meleg, I., Iepure, S., Povară, I. (2011). Spatial distribution patterns of the hyporheic invertebrate communities in a polluted river in Romania. Hydrobiologia, 669, 63-82.

Motaş, C. (1923). Contribution à l'étude des Acariens d'eau douce de Roumanie. Ann. sci. Univ. Jassy, $12(3-4), 261-275$.

Motaş, C. (1940). Un Arrenurus nouveau trouvé dans les environs de Jassy. Hommage à la Mém. du Prof. Al. Slatineanu, Jassy, $1-7$.

Motaş, C. (1959). Descriera a trei Hidracarieni noi. Acad. Republ. Pop. Rom. Omagiu lui Traian Savulescu cu prilejul împlinirii a 70 de ani.

Motaş, C., Șoarec, J. (1939). Sur deux Feltria nouvelles trouvées dans les Carpates Orientales et sur les caractères sexuels secondaires dans le genre Feltria Koen. 1892. Memoriile Secțiunii Științifice, Academia Română, București, 15 (2), 29-43.

Motaş, C., Tanasachi, J. (1944). Hydracariens des Carpates Roumaines (Massif de Bucegi et Alpes de Transylvanie). Memoriile Secțiunii Științifice, 19 (7), 241-256.

Motaş, C., Tanasachi, J. (1946). Acariens phréaticoles de Transylvanie. Notat. biolog., 4 (1-3), 1-63.

Motaş, C., Tanasachi, J. (1948a). Diagnoses de trois nouvelles Hydrachnelles phréaticoles de Roumanie. Ann. scient. Univ. Jassy, 31, 146-151.

Motaş, C., Tanasachi, J. (1948b). Espèces nouvelles et connues du genre Megapus Neuman (Hydrachnelles) trouvées dans les eaux souterraines. Ann. scient. Univ. Jassy, 31, 152-169. 
Motaş, C., Tanasachi, J. (1960). Une nouvelle espèce phréaticole du genre Atractides Koch (Acari) recueillie dans la Région autonome Magyare (R. P. Roumaine). Vestnuk Ceskoslov. Zool., Spol., 24 (4), 342-345.

Motaş, C., Tanasachi, J. (1963). Hydrachnellae freatice din bazinul Vîrghisului. (Hydrachnellae phréatiques du bassin du Vîrghis Région de Mureș-Autonome Hongroise). Lucrările Institutului de Speologie «E. Racovitza», 1-2 (1962-1963), 311-340.

Motaş, C., Tanasachi, J., Orghidan, T. (1946). Un Hydracarien nouveau de la Roumanie: Frontipodopsis transylvanica n. sp. Bull. sect. scient. Acad. Roumaine., 29 (1), 29-34.

Motaş, C., Tanasachi, J., Orghidan, T. (1947a). Un nouveau Hydracarien phréaticole recueilli en Transylvanie. Bull. sect. scient. Acad. Roumaine, 29 (5), 303-307.

Motaş, C., Tanasachi, J., Orghidan, T. (1947b). Diagnoses de quelques nouveaux Hydracariens phréaticoles de Roumanie. Bull. sect. scient. Acad. Roumaine, 29 (8), 506-512.

Motaş, C., Tanasachi, J., Orghidan, T. (1947c). Hydracariens phréaticoles de Roumanie. Notat. biolog., București, 5 (1-3), 1-67.

Motaş, C., Tanasachi, J., Orghidan, T. (1957a). Über einige neue phreatische Hydrachnellae und über Phreatobiologie, ein neues Kapitel der Limnologie. Abh. naturw. Ver. Bremen, 35 (1), 101-122.

Motaş, C., Tanasachi, J., Orghidan, T. (1957b). Sur un hydracarien nouveau de la sous-famille des Thyasinae (Placothyas fibulata n. g. n. sp.) et sur l'homologie des formations cuticulaires dans ce groupe. Ark. Zool., 2 (11-12), 167-185.

Motaş, C., Tanasachi, J., Orghidan, T. (1958). Hydrachnelles phréaticoles de la R. P. Roumaine. Vestnik Ceskolov. Zool. Spol., 22 (4), 293-333.

Pavelescu, C., Cîmpean, M. (2002-2003). Preliminary study on oligochaetes (Annelida, Oligochaeta) and water mites (Acari, Hydrachnidia) from hyporheic zones of the Crişul Repede River (Romania). Travaux de l'Institut de Spéologie "É. Racovitza", 41-42, 149-158.

Șoarec, J. (1939). Contributie la studiul Hidracarienilor din România-Carpații orientali. Memoriile Secțiunii Științifice, Academia Română (Seria III) 14, 363-373.

Tanasachi, J., Orghidan, T. (1955). Hidracarieni orbi din apele freatice. Bul. Știint., Sect. Biol., Agronom., Geol., Geogr., 7 (2), 369-381.

Viets, K.O. (1978). Hydracarina. In: Illieș, J. (ed.), Limnofauna Europaea (pp. 154-181).

Watermite.org. 2017. European Water mite Research - A website informing on water mite research in Europe. Retrieved from: http://www.watermite.org.

Cite as: Cîmpean, M., Battes, K.P. (2018). Present status of water mite species (Acari, Hydrachnidia) described by Motaş, Tanasachi and Orghidan from Romania. Acta Biologica, 25, 59-68. DOI: 10.18276/ ab.2018.25-05. 